\title{
Water Stress Plating Hypersensitivity of Yeasts: Protective Role of Trehalose in Saccharomyces cerevisiae
}

\author{
By KYLIE F. MACKENZIE, $\dagger$ K. K. SINGH AND A. D. BROWN* \\ Department of Biology, University of Wollongong, Wollongong, NSW 2500, Australia
}

(Received 5 August 1987; revised 24 November 1987)

\begin{abstract}
Water stress plating hypersensitivity was studied in two strains of Saccharomyces cerevisiae, one of them being a mutant incapable of accumulating trehalose to significant levels. The wild-type strain was grown in a defined medium with glucose, maltose or ethanol as carbon/energy source. In each case plating hypersensitivity was demonstrated and resistance to the stress developed in the second half of the exponential growth phase. Development of resistance was accompanied by accumulation of trehalose and was apparently unrelated to glycerol content which, under these conditions, was always low. A qualitatively similar trend was observed in the mutant grown on glucose but trehalose levels remained low and recovery of stress resistance was only slight. Dinitrophenol induced trehalose breakdown in resting yeast and simultaneously induced the onset of plating hypersensitivity. A negative correlation was demonstrated between trehalose content and 'plating discrepancy' (log colony count on 'normal' agar - log colony count on stressing agar) for both strains under all experimental conditions. The correlation held for trehalose contents up to about $50 \mathrm{mg}$ (g dry yeast) ${ }^{-1}$, above which the yeasts were apparently fully resistant. Trehalose was evidently a more effective compatible solute, per mole, than glycerol.
\end{abstract}

\section{INTRODUCTION}

The phenomenon that we have called 'water stress plating hypersensitivity' (Brown et al., 1986; Mackenzie et al., 1986) is demonstrated by viable counts during growth of a sensitive yeast such as Saccharomyces cerevisiae in a conventional high water activity $\left(a_{\mathrm{w}}\right)$ liquid growth medium. When plate counts are made on both a high $a_{\mathrm{w}}$ nutrient agar medium and one of low $a_{\mathrm{w}}$ such as 'synthetic honey agar' (containing $48 \%, \mathrm{w} / \mathrm{v}$, glucose), there is a discrepancy of some 3-4 logarithms between the counts on the two media in mid-exponential phase. In stationary phase the discrepancy diminishes, ultimately and usually to insignificance. The magnitude of the plating discrepancy is a function of the solute concentration (or $a_{\mathrm{w}}$ ) of the low $a_{\mathrm{w}}$ plating medium. At solute concentrations above a critical threshold value, the discrepancy is roughly proportional to the solute concentration; below the threshold there is no discrepancy (Mackenzie et al., 1986).

Xerotolerant yeasts do not have the characteristic nor do some others that would not normally be considered xerotolerant. Plating hypersensitivity was found in only three of fourteen species and strains of yeasts screened. All resistant strains accumulated significant quantities of at least one polyhydric alcohol while growing exponentially in a conventional high $a_{\mathrm{w}}$ broth. Sensitive strains did not and we concluded from this and other evidence that the compatible solute properties of the polyols were sufficient to protect against the potentially lethal effects of the sudden dehydration incurred by plating into a stressing agar medium (Mackenzie $e t$ al., 1986).

† Present address: Department of Microbiology and Cell Science, Institute of Food and Agricultural Sciences, University of Florida, Gainsville, Florida 32611, USA.

Abbreviations: BYM, basal yeast medium; MA, malt-extract agar; SHA, synthetic honey agar. 
Protection by polyols, however, did not explain the recovery of resistance by sensitive strains on entering stationary phase of a growth cycle. Many yeasts and fungi, when grown in laboratory batch culture, accumulate trehalose as the culture ages; the accumulation is a consequence of a change in the dynamics and regulation of carbohydrate metabolism (Küenzi \& Fiechter, 1972; Fiechter et al., 1981; Thevelein, 1984a). Our strain of $S$. cerevisiae is no exception and we have already presented some evidence that trehalose confers resistance against a sudden severe solute stress (Brown et al., 1986). The present paper adds to that evidence.

\section{METHODS}

Organisms. Saccharomyces cerevisiae (strain Y41, ATCC 38531) and S. cerevisiae (strain 212-244-1A) (Yeast Genetic Stock Center, University of California, Berkeley, USA) were maintained and cultured according to Edgley \& Brown (1983); they were counted according to Mackenzie et al. (1986). Two liquid culture media were used, namely basal yeast medium (BYM, a conventional broth, $0.997 a_{\mathrm{w}}, \psi_{\pi}=-0.42 \mathrm{MPa}$ at $30^{\circ} \mathrm{C}$ ), and Wickerham's synthetic medium (Barnett et al., 1983), of approximately the same $a_{\mathrm{w}}$. This medium, without the carbon source. Samples $(200 \mathrm{ml})$ were filtered aseptically through a membrane $(0.8 \mu \mathrm{m}$ pore size) and then before use. The carbon source was mostly used at a final concentration of $0.5 \%(w / v)$. The plating media used were malt extract agar (MA, conventional complex high $a_{\mathrm{w}}$ medium) and 'synthetic honey agar' (SHA, 48\%, w/v, glucose, $a_{\mathrm{w}} 0.924, \psi_{\pi}=-11 \cdot 1 \mathrm{MPa}$ at $30^{\circ} \mathrm{C}$; Anand \& Brown, 1968). In experiments in which strain Y41 was exposed to dinitrophenol (DNP), the yeast was first grown for $72 \mathrm{~h}$ in Wickerham's medium with glucose as the carbon source. Samples $(200 \mathrm{ml})$ were filtered aseptically through a membrane $(0.8 \mu \mathrm{m}$ pore size $)$ and then transferred, with the filter, to fresh synthetic medium $(200 \mathrm{ml})$ without a carbon source, with and without DNP (2 mM).

Analytical methods. Samples $(10 \mathrm{ml})$ of culture suspension were withdrawn and filtered rapidly under vacuum through a cellulose acetate membrane filter $(0.8 \mu \mathrm{m}$ pore size). If trehalose were to be estimated in yeast sampled from media containing either glucose or maltose, the yeast was washed twice on the filter with potassium phosphate buffer (pH 5.9), isotonic with the growth medium. Otherwise the yeast was not washed. The yeast was then frozen in liquid nitrogen, freeze-dried and stored at $-80^{\circ} \mathrm{C}$ pending extraction. Filtrates were also frozen in liquid nitrogen and stored at $-80^{\circ} \mathrm{C}$ for periods of up to 4 weeks until analysed. Freeze-dried yeast was extracted with ethanol according to Edgley \& Brown (1983). For comparative purposes trehalose was also estimated in trichloroacetic acid $(0.5 \mathrm{M})$ extracts (Trevelyan \& Harrison, 1952) but there was no significant difference between the two methods of extraction. Approximately $1 \mathrm{ml}$ ethanol or TCA solution was used per $4 \mathrm{mg}$ dry yeast. Ethanolic extracts were dried under vacuum, redissolved in water $(1.0 \mathrm{ml})$ and stored at $-80^{\circ} \mathrm{C}$. Trichloroacetic acid extracts were treated according to Trevelyan \& Harrison (1952), adjusted to $1.0 \mathrm{ml}$ and stored at $-80^{\circ} \mathrm{C}$.

Ethanol was estimated (in filtrates only) according to Beutler (1984). Glucose and glycerol were estimated as described previously (Edgley \& Brown, 1983). Trehalose was estimated by reaction with anthrone (Stewart, 1975) in $0.2 \mathrm{ml}$ volumes of cell extract.

\section{RESULTS AND DISCUSSION}

When $S$. cerevisiae was grown at $15^{\circ} \mathrm{C}$ in BYM it had essentially the same characteristics of plating hypersensitivity (not illustrated) as described previously (Mackenzie et al., 1986) for growth at $30{ }^{\circ} \mathrm{C}$. This contrasts with the correlation previously observed (Mackenzie et al., 1986) between nutrient-limited growth rate in continuous culture and plating discrepancy (log MA plate count $-\log$ low $a_{\mathrm{w}}$ plate count). Evidently, survival of exponential phase yeast after a sudden solute stress is affected not simply by growth rate itself but rather by the factors that limit growth rate. Nutrient limitation in continuous culture, like the cessation of exponential growth in batch culture, is effective whereas a general suppression of all reaction rates is not.

Growth at $30^{\circ} \mathrm{C}$ in synthetic medium with glucose, maltose or ethanol as carbon source all gave growth curves with typical water stress plating hypersensitivity. Fig. 1 shows results of a series of assays on $S$. cerevisiae (Y41) grown with glucose as the carbon source. Exhaustion of glucose, the start of measurable consumption of ethanol, the maximum in the proportion of budded yeast cells and the minimum in the SHA plate count all happened within 4-6 h of inoculation. Both trehalose and glycerol content fell initially; trehalose reached an apparent minimum about $8 \mathrm{~h}$ after inoculation. Essentially similar results were obtained when either ethanol or maltose was the carbon source even though maltose, in contrast to glucose, has been reported to sustain trehalose accumulation during exponential growth (Panek et al., 1978).

When stationary phase yeast is transferred to fresh medium, the accumulated trehalose is 


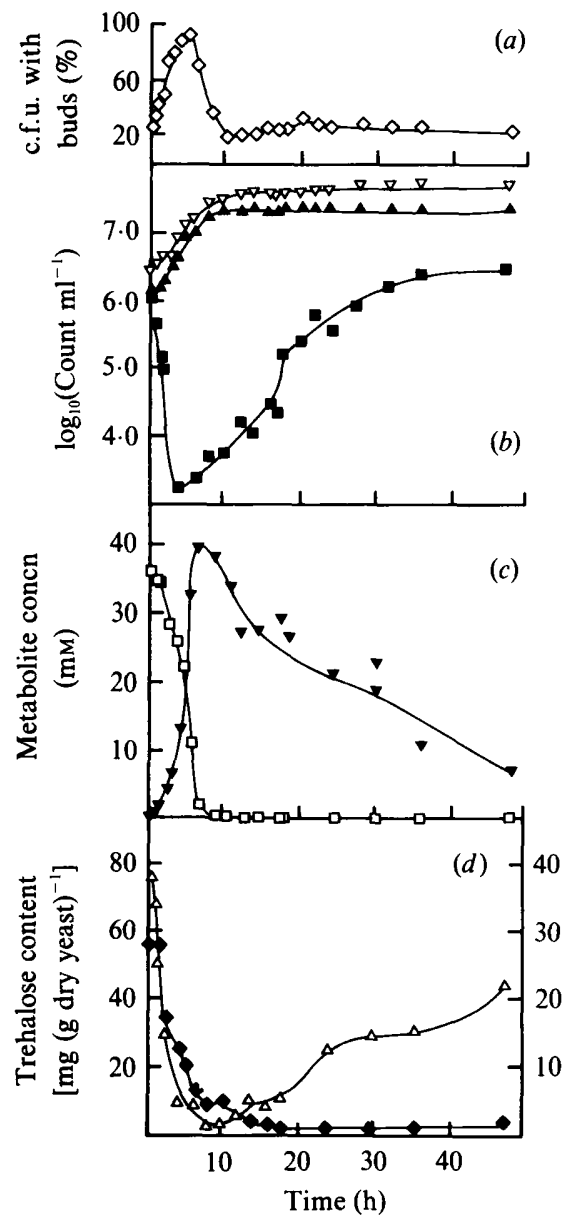

Fig. 1

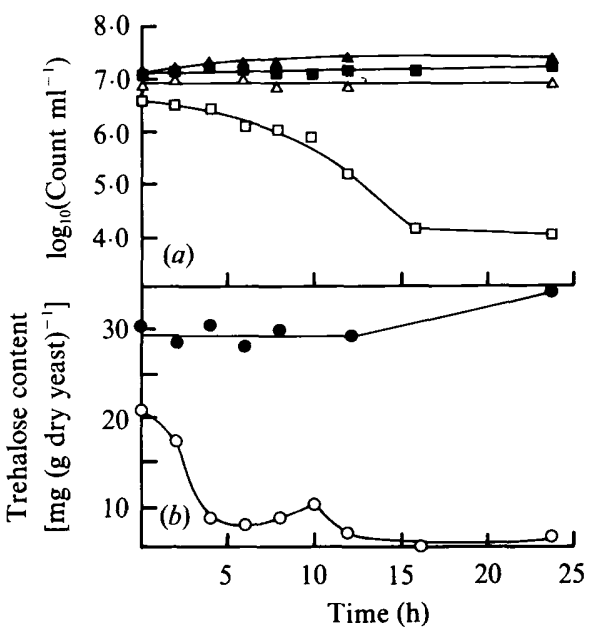

Fig. 2

Fig. 1. Partial growth cycle of $S$. cerevisiae strain Y41 on synthetic medium with glucose as carbon/energy source. (a) Proportion of c.f.u. with buds. (b) $\nabla$, Total count; $\Delta$, MA viable count; $\square$, SHA viable count. (c) Glucose $(\square)$ and ethanol $(\nabla)$ concentration in the medium. (A corresponding plot for glycerol concentration had essentially the same shape as that for ethanol but with a maximal glycerol concentration of about $2.5 \mathrm{~mm}$.) (d) Intracellular glycerol $(\bullet)$ and trehalose $(\triangle)$. Points represent the means of two separate experiments; in $(b)$ the means of the logarithms are used.

Fig. 2. Effect of DNP (2.0 mM) on the viability, water stress plating hypersensitivity and trehalose content of resting $S$. cerevisiae strain Y41 suspended in synthetic medium without a carbon/energy source. (a), Viable counts: $\Delta, \mathbf{a}$, MA colony counts; $\triangle, \square$, SHA colony counts. $\Delta, \triangle$, Suspensions without DNP; $\square, \square$, suspensions with DNP. (b) Trehalose content : $\bigcirc$, suspension without DNP; $\bigcirc$, suspension with DNP.

rapidly hydrolysed by trehalase, which is activated by glucose metabolism. The mechanism usually ascribed to the activation process involves phosphorylation of the enzyme protein by cyclic AMP (e.g. Thevelein \& Beullens, 1985).

Membrane depolarizing agents such as DNP stimulate trehalose breakdown in yeast; this has also been attributed to activation of trehalase by phosphorylation (Thevelein, 1984b). On the other hand, Valle et al. (1986) have presented evidence that activation of trehalase can be attributed to acidification of the cytoplasm rather than to membrane depolarization. Whatever the mechanism, however, there is ample empirical evidence that DNP stimulates the breakdown of trehalose in resting yeast. Fig. 2 shows that exposure of strain Y41 to DNP did not 


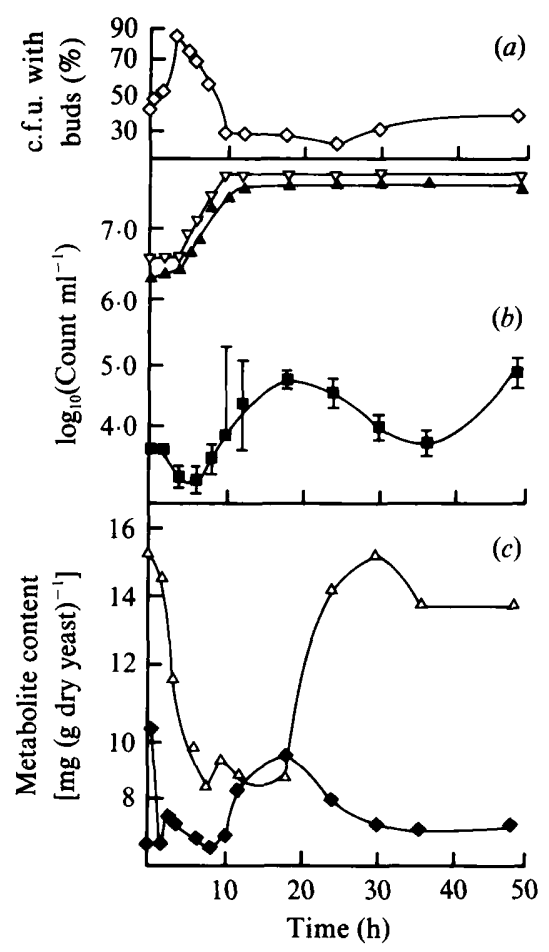

Fig. 3.

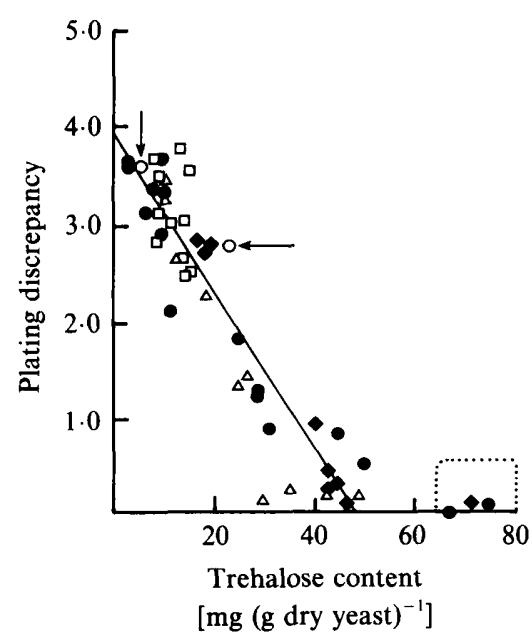

Fig. 4.

Fig. 3. Partial growth cycle of $S$. cerevisiae strain 212-244-1A (glcl, trehalose negative) on synthetic medium with glucose as carbon/energy source. ( $a$ ) Proportion of c.f.u. with buds. $(b) \nabla$, Total count; $\boldsymbol{A}$, MA viable count; $\square$, SHA viable count. (c) Intracellular glycerol $(\diamond)$ and trehalose $(\triangle)$. Points represent the means and SD of three separate experiments; in (b) the mean of the logarithm is used. Where error bars are not shown the SD lay within the symbols. Note the difference in the trehalose scale between this Figure and Fig. 1.

Fig. 4. Plating discrepancy $v$ s trehalose content for strain Y41 grown in synthetic medium on glucose $(\bigcirc)$, maltose $(\triangle)$ and ethanol $(\diamond)$ and strain 212-244-1A grown on glucose $(\square)$. The three boxed-in points were excluded from the calculation of the regression as were the two open circles (arrowed) which denote values for strain Y41 at two different dilution rates in complex medium in continuous culture. The regression line has a slope of -0.084 and a correlation coefficient of -0.94 . Other interpretations are possible (see text).

affect viability on MA but did promote a decrease in both viability on SHA and trehalose content, although there were differences in the patterns of change.

Additional evidence for a protective role of trehalose was provided by strain 212-244-1A, which carries the glcl mutation, a regulatory pleiotropic mutation that diminishes the accumulation of both trehalose and glycogen. Trehalose accumulation is prevented because trehalase always remains in its phosphorylated active form to a significant extent (Ortiz et al., 1983; Tenan et al., 1985). We reported previously preliminary results indicating that strain 212244-1A grown in complex medium does not recover from its hypersensitive state on entering stationary phase (Brown et al., 1986). Fig. 3 shows results of a similar but more comprehensive experiment in which the glcl mutant strain was grown throughout in synthetic medium.

The total count rose to a slightly higher level than did that of strain Y41 in similar circumstances (Fig. 1) but, although the SHA count oscillated throughout the growth cycle, it did not recover in stationary phase; the plating discrepancy remained between 2.9 and 3.9 throughout the growth cycle. The concentrations of glucose, ethanol and glycerol in the medium were similar to those produced by Y41 (Fig. 1) and are not illustrated. The changes in intracellular glycerol content, however, were different from those encountered in strain Y41 
(Fig. 1). There was a peak in glycerol content after 15-20 h when the organism had entered stationary phase. This corresponded closely with a peak in the SHA plate count. There was no such peak with Y41 although there was some evidence of a small shoulder after about $10 \mathrm{~h}$. Moreover, although the maximal glycerol content was higher in Y41, glycerol content remained higher in the glcl strain late in the growth cycle.

The pattern of trehalose content throughout the growth cycle was similar to that of Y41 inasmuch as there was a minimum after about $5 \mathrm{~h}$ (Fig. 3) but the subsequent course of accumulation was different in the two strains. The highest value encountered in 212-244-1A was slightly over $0.042 \mathrm{mmol}$ (g dry yeast) $)^{-1}(1.5 \%)$ compared with $0.21 \mathrm{mmol} \mathrm{g}^{-1}(7.5 \%)$ for Y41.

The quantitative relation between trehalose content and plating discrepancy is shown in Fig. 4 for both strain 212-244-1 A grown on glucose and strain Y41 grown separately on glucose, maltose and ethanol. An alternative interpretation of these results is that the relation between plating discrepancy and trehalose content is curved. A linear regression with a correlation coefficient of -0.91 can be fitted to a plot of plating discrepancy $v s \log$ trehalose content (with the boxed points included). (The inclusion of the boxed points in the arithmetic relation numerically lowers the correlation coefficient to -0.90.) From theoretical considerations, however, we consider that Fig. 4 represents the most likely quantitative interpretation of the results. It implies a negative linear relation between plating discrepancy and mean trehalose content up to a value of about $50 \mathrm{mg}$ ( $\mathrm{g}$ dry yeast $)^{-1}$. At higher levels, the entire population is apparently resistant.

For mean trehalose contents of less than $50 \mathrm{mg} \mathrm{g}^{-1}$, the mathematical significance of Fig. 4 is as follows

$$
\triangle P D=-0.084 \Delta T
$$

where $P D$ denotes plating discrepancy and $T$ the trehalose content of the yeast. By definition, $P D=\log M-\log S$, where $M$ is the MA plate count and $S$ the SHA plate count. Since $M$ is independent of trehalose content, $\Delta \log M=0$ and therefore

$$
-\Delta \log S=-0.084 \Delta T
$$

or

$$
\Delta \log S=0.084 \Delta T
$$

This equation will not be valid for a stress of a magnitude different from that used in these experiments and might not be valid for different culture conditions. Nevertheless, the values obtained from a complex medium in continuous culture lay close to the regression line (Fig. 4).

In practical terms, equation (2) means that a change of $1 \mathrm{mg} \mathrm{g}^{-1}$ in the mean trehalose content of a yeast population will be accompanied by a change in the number of survivors on SHA of 0.084 logarithms ( 1.2 fold). The trehalose analyses, however, represent mean values for the bulk population whereas the plate counts, which are digital measurements, divide the population into two groups, viable and non-viable. If trehalose is, in fact, the principal protective agent then it would be expected to be distributed non-uniformly in the population; for our immediate purpose, its distribution can be expressed as values either above or below a critical threshold level. Fig. 4 indicates that the entire population was protected at a minimum mean value of about $50 \mathrm{mg}$ trehalose ( $\mathrm{g}$ dry yeast) ${ }^{-1}$. At levels higher than this, the population was demonstrably homogeneous with respect to stress resistance and therefore we can assume that, under such conditions, trehalose is more uniformly distributed than it is in the linearly responsive part of Fig. 4. Hence $50 \mathrm{mg} \mathrm{g}^{-1}$ (or $0.15 \mathrm{mmol} \mathrm{g}^{-1}$ ) should approximate the critical threshold value for a single yeast cell. Under these conditions trehalose is thus a more effective compatible solute than glycerol which confers complete protection at about $2 \mathrm{mmol}$ (g dry yeast) ${ }^{-1}$ (Mackenzie et al., 1986). Micro-organisms generally are more resistant to chemical and physical stresses when resting than when multiplying rapidly. It is unlikely that trehalose is the only source of resistance to solute stress in stationary phase yeast, even though there is no evidence from our results for a major direct contribution by any other mechanism.

Trehalose has also been reported to accumulate in $S$. cerevisiae in response to heat under which conditions it protects against both heat shock and desiccation (Hottiger et al., 1987). All 
compatible solutes might not have the same mode of action, however. For example, trehalose is especially effective in stabilizing lipoprotein membranes and lipid monolayers (Crowe et al., $1984 a, b$ ), whereas glycerol is notable for protecting soluble enzymes (see Brown, 1978).

Trehalose has been reported to be an osmoregulatory solute in some prokaryotes (e.g. MacKay et al., 1984; Strøm et al., 1986; Larsen et al., 1987); it necessarily contributes to the overall osmotic status of yeast but it is far from clear that it has an osmoregulatory function. Edgley (1980) reported an increase in trehalose content of $S$. cerevisiae grown at an elevated salt concentration, but the dependence of trehalose accumulation on a low energy flux is sufficient to deny it a significant role as an osmoregulator in a strict sense.

\section{REFERENCES}

ANAND, J. C. \& Brown, A. D. (1968). Growth rate patterns of the so-called osmophilic and nonosmophilic yeasts in solutions of polyethylene glycol. Journal of General Microbiology 52, 205-212.

Barnett, J. A., Payne, R. W. \& Yarrow, D. (1983). Yeasts: Characteristics and Identification. Cambridge: Cambridge University Press.

BeUTLER, H. O. (1984). Ethanol. In Methods of Enzymatic Analysis, pp. 598-606. Edited by H. U. Bergmeyer, J. Bergmeyer \& M. Grassl. Weinheim: Verlag Chemie.

Brown, A. D. (1978). Compatible solutes and extreme water stress in eukaryotic micro-organisms. $A d$ vances in Microbial Physiology 17, 181-242.

Brown, A. D., Mackenzie, K. F. \& Singh, K. K. (1986). Selected aspects of microbial osmoregulation. FEMS Microbiology Reviews 39, 31-36.

Crowe, L. M., Mouradian, R., Crowe, J. H., JACKSON, S. A. \& WOMERSLEY, C. (1984a). Effects of carbohydrates on membrane stability at low water activities. Biochimica et biophysica acta 769, 141-150.

Crowe, J. H., Whittam, M. A., Chapman, D. \& CROWE, L. M. (1984b). Interaction of phospholipid monolayers with carbohydrates. Biochimica et biophysica acta 769, 151-159.

EDGLEY, M. (1980). Osmoregulation in xerotolerant and non-tolerant yeasts. PhD thesis, University of Wollongong, NSW, Australia.

EDGley, M. \& Brown, A. D. (1983). Yeast water relations: physiological changes induced by solute stress in Saccharomyces cerevisiae and Saccharomyces rouxii. Journal of General Microbiology 129, 34533463.

Fiechter, A., Fuhrman, G. F. \& Käppeli, O. (1981). Regulation of glucose metabolism in growing yeast cells. Advances in Microbial Physiology 22, 123-183.

Hottiger, T., Boller, T., \& Wiemken, A. (1987). Rapid changes of heat and desiccation tolerance correlated with changes of trehalose content in Saccharomyces cerevisiae cells subjected to temperature shifts. FEBS Letters 220, 113-115.

KÜENZI, M. T. \& FIECHTER, A. (1972). Regulation of carbohydrate composition of Saccharomyces cerevisiae under growth limitation. Archiv für Mikrobiologie 84, 254-265.

LaRsen, P. I., Sydnes, L. K., Landfald, B. \& Strøm, A. R. (1987). Osmoregulation in Escherichia coli by accumulation of organic osmolytes: betaines, glutamic acid and trehalose. Archives of Microbiology 147, 1-7.

MacKay, M. A., Norton, R. S. \& Borowitzka, L. J. (1984). Organic osmoregulatory solutes in cyano- bacteria. Journal of General Microbiology 130, 2177 2191.

Mackenzie, K. F., Blomberg, A. \& Brown, A. D. (1986). Water stress plating hypersensitivity of yeasts. Journal of General Microbiology 132, 20532056.

Ortiz, C. H., Maia, J. C. C., Tenan, M. N., BrazPadrao, G. R., Mattoon, J. R. \& Panek, A. D. (1983). Regulation of yeast trehalase by a monocyclic, cyclic AMP-dependent phosphorylation-dephosphorylation cascade system. Journal of Bacteriology 153, 644-651.

Panek, A. D., Sampaio, A. L., Braz, G. C. \& Mattoon, J. R. (1978). Regulation of energy metabolism in yeast. Relationships between carbohydrate reserves, catabolite repression and maltose utilization. In Biochemistry and Genetics of Yeasts. Pure and Applied Aspects, pp. 145-160. Edited by M. Bacila, B. L. Horecker \& A. O. M. Stoppani. New York: Academic Press.

StewART, P. R. (1975). Analytical methods for yeasts. Methods in Cell Biology 12, 111-147.

Strøm, A. R., Falkenberg, P. \& LANDFALd, B. (1986). Genetics of osmoregulation in Escherichia coli: uptake and biosynthesis of organic osmolytes. FEMS Microbiology Reviews 39, 79-86.

Tenan, M. N., Ortiz, C. H., Dellamora-Ortiz, G. M., Mattoon, J. R. \& Panek, A. D. (1985). Relationships between mutations affecting protein kinase and accumulation of energy reserves in Saccharomyces cerevisiae. FEMS Microbiology Letters 26, 217-220.

Thevelein, J. M. (1984a). Regulation of trehalose mobilization in fungi. Microbiological Reviews 48, 42-59.

Thevelein, J. M (1984b). Activation of trehalase by membrane-depolarizing agents in yeast vegetative cells and ascospores. Journal of Bacteriology. 158, 337-339.

Thevelein, J. M. \& Beullens, M. (1985). Cyclic AMP and the stimulation of trehalase activity in the yeast Saccharomyces cerevisiae by carbon sources, nitrogen sources and inhibitors of protein synthesis. Journal of General Microbiology 131, 3199-3209.

Trevelyan, W. E. \& Harrison, J. S. (1952). Studies on yeast metabolism. 1. Fractionation and microdetermination of cell carbohydrates. Biochemical Journal 50, 298-302.

Valle, E., Bergillos, L., Gascón, S., Parra, F. \& RAMOS, S. (1986). Trehalase activation in yeasts is mediated by an internal acidification. European Journal of Biochemistry 154, 247-251. 